\title{
Zinc finger A20/AN1 stress-associated genes, $H v S A P$, are differentially expressed under drought, salinity and dehydration in barley leaves
}

\author{
Baidyussen A. ${ }^{1 *}$, Jatayev S. ${ }^{1}$, Kurishbayev A. ${ }^{1}$, Langridge P. ${ }^{2,3}$, Schramm C. ${ }^{4}$, \\ Jenkins C. ${ }^{4}$, Soole K. ${ }^{4}$, Shavrukov Y. ${ }^{4}$ \\ ${ }^{1}$ Faculty of Agronomy, S. Seifullin Kazakh AgroTechnical University, Nur-Sultan, Kazakhstan \\ ${ }^{2}$ Wheat Initiative, Julius Kühn-Institute, Berlin, Germany \\ ${ }^{3}$ University of Adelaide, $S A$, Australia \\ ${ }^{4}$ College of Science and Engineering, Biological Sciences, Flinders University, SA, Australia \\ *e-mail:bai_akmaral@mail.ru
}

Introduction and Aim: A family of genes designated the Zinc fingerA20/AN1Transcription factors, encoding stress-associated proteins $(S A P)$, represent a large group of genes in both plants and animals. The gene family, which includes $14 A t S A P$ and 18 OsSAPgenes, is well described in Arabidopsis and rice, where variable tolerance to multiple abiotic stresses were studied and a great diversity in structure and function of the $S A P$ gene family was found in different plant species. The aim of this study was to identify all HvSAP genes in barley (Hordeum vulgare L.) and to carry out experiments determining gene expression in response to drought, salinity and dehydration in barley leaves.

Methods: Bioinformatic approaches were used to identify all HvSAP genes in barley and construct a molecular phylogenetic tree using publicly available databases and computer software. For gene expression, qPCR analysis was carried out on cDNA synthesized from mRNA extracted from control and treated barley plants.

Results: In our study with barley, 17 HvSAP genes were most commonly identified, which were strongly homologous to rice genes. Small rearrangements inthe barley genome were found, where two HvSAP genes were duplicated, but three other genes were lost compared to the $18 O S S A P$ genes in rice. Multiple and quite variable responses in $H v S A P$ gene expressions were found in treated barley plants compared to controls, where some but not all gene expression profiles were similar to those published in rice. Repeated experiments on $H v S A P$ gene expression in response to drought, salinity and dehydration will verify the confidence of our results in barley in the nearest future.

Acknowledgements: This study was supported by the Ministry of Education and Science, Kazakhstan, Research program BR05236500 (SJ). 